\title{
Periodontal Health Awareness among IIUM Medical Students
}

Suhaila Muhammad Ali, Nurdayana Khairul Anuar, Nurul Najah Abu Bakar, Munirah Yaacob, Juzaily Husain

Kulliyyah of Dentistry, International Islamic University Malaysia, Kuantan, Pahang, Malaysia

Introductions: Proper knowledge of oral diseases is crucial in medical practice because periodontal disease is associated with multiple systemic conditions. A large number of systemic diseases and drugs have oral manifestations as well as adverse reactions. The aim of this study is to assess periodontal awareness, knowledge and attitude among medical students of International Islamic University Malaysia (IIUM). Materials and method: This cross-sectional study utilized questionnaires which were distributed to fourth and final year medical students of IIUM Kuantan. Each participant's consent was obtained before being recruited into the survey. Questions pertaining to oral health, periodontal disease and its relation with systemic conditions were given. Based on the answers, respondents' awareness, knowledge and attitude were assessed. The data was analyzed by using Statistical Package for Social Sciences (SPSS) version 21. Results: A total of 93 students completed the survey questionnaires. $3 \%$ of them have awareness that periodontal disease is related to gingiva. $10 \%$ respondents agreed that the dental plaque is the primary cause of periodontal disease.80\% respondents have knowledge of associations between periodontal disease and systemic conditions. $4 \%$ of them were able to identify drugs causing gingival enlargement. $97 \%$ of respondents showed positive attitude for referral to dentist in the future. Conclusions: The participants have superficial awareness and knowledge about periodontal disease and its relation to systemic conditions. However, they have positive attitude for future practice towards their patients. Hopefully, this research may provide direct suggestion to incorporate basic periodontal knowledge in their medical syllabus. 